\title{
latrogenic phrenic nerve palsy
}

\section{Jennifer Whiteley, Mohamed Shoeib, Rocco Bilancia}

Department of Cardiothoracic Surgery, Golden Jubilee National Hospital, Glasgow, UK

Contributions: (I) Conception and design: All authors; (II) Administrative support: All authors; (III) Provision of study materials or patients: All authors; (IV) Collection and assembly of data: All authors; (V) Data analysis and interpretation: All authors; (VI) Manuscript writing: All authors; (VII) Final approval of manuscript: All authors.

Correspondence to: Rocco Bilancia. Department of Cardiothoracic Surgery, Golden Jubilee National Hospital, Agamemnon Street, Clydebank, G81 4DY, Glasgow, UK. Email: rocco.bilancia@gjnh.scot.nhs.uk.

\begin{abstract}
Iatrogenic phrenic nerve injury is an issue that all thoracic surgeons will be called to tackle during their career. Injury of the phrenic nerve leads to paralysis of the hemidiaphragm, which can result in a spectrum of symptom severity from asymptomatic to disabling shortness of breath. It can result from any operation requiring dissection close to its anatomical pathway from the neck to the diaphragm, but is also at risk with interventional procedures within its vicinity, such as cardiac ablation techniques. Injury can range from complete, irreversible transection of the nerve to neuropraxia with temporary nerve dysfunction. Due to this the timing of intervention is individual to each patient. Symptoms may range from mild to severe and from typical to aspecific. Confirmation of diagnosis is accomplished with a combination of static (CXR, CT) and dynamic imaging (ultrasound, fluoroscopy). The presence of other co-morbidities such as obesity, heart conditions or obstructive airways disease potentially contributing to the same symptoms, makes relative contribution of each difficult to ascertain and may increase surgical risks. A careful risk/benefit assessment, as well as the optimisation of these underlying conditions prior to treatment is paramount. Treatment options include minimally invasive diaphragmatic plication as well as nerve repair techniques. We hereby give an overview of the relevant anatomy and aetiology of iatrogenic phrenic nerve injury, as well as of the diagnostic work-up and different therapeutic options, with a focus on diaphragmatic plication, nerve repair and treatment timing.
\end{abstract}

Keywords: Iatrogenic phrenic nerve injury; diaphragm plication; video-assisted surgery plication (VATS plication); phrenic nerve repair

Received: 14 February 2020; Accepted: 08 July 2020; Published: 10 July 2021.

doi: 10.21037/shc-2019-amp-01

View this article at: http://dx.doi.org/10.21037/shc-2019-amp-01

\section{Introduction}

Phrenic nerve injury can occur as a result of many different causes, including iatrogenic injury. This results in paralysis of the hemidiaphragm on the affected side and patients can experience a range of severity of symptoms especially in the presence of other co-morbidities. Depending on the nature of the injury, some may spontaneously recover, whereas some mechanisms of injury can lead to permanent paralysis, with gradual potentially debilitating consequences. Here we discuss the anatomy and aetiology of iatrogenic phrenic nerve injury, as well as diagnostic work up and evidence and timing for therapeutic options.

\section{Surgical anatomy}

The phrenic nerves, are formed in the neck, arising from the ventral ramus of $\mathrm{C} 4$ with contributions from $\mathrm{C} 3$ and $\mathrm{C} 5$ nerve roots at the lateral border of the scalene muscles. The right phrenic nerve crosses over the right mammary artery at its origin and follows the course of the right innominate vein and superior vena cava as it descends into the chest. On the left, at the thoracic inlet, the phrenic is related anteriorly to thoracic duct and posteriorly to subclavian 
artery. Then, it passes anterior to mammary artery and descends to lie in between the left subclavian and common carotid arteries, crossing in front of the corresponding vagus nerve. Thereafter, it crosses lateral on the left side of the aortic arch. Bilaterally, the phrenic nerves pass anterior to the hilum of the lung above the pericardium; at the level of the corresponding diaphragm, both phrenic nerves then divide into multiple terminal branches (1).

Accessory phrenic nerves are small branches that arise from the subclavian nerves and are present in $20-30 \%$ of normal people. They merge the main phrenic nerves either at the base of the neck or at the thoracic inlet. When they exist, if the phrenic nerves are damaged above the level of the accessory phrenic nerves, they may provide some motor function to the corresponding hemidiaphragm (2).

The phrenic nerves deliver motor supply to the diaphragm, which is the primary muscle of respiration. Contraction of its fibres causes a downward movement that generates negative intrathoracic pressure and subsequent air entry and lung expansion. If the diaphragm is denervated, it becomes atonic and floppy as a result of gradual atrophic processes. In addition to the obvious loss of breathing function and dynamic efficiency on the affected side, paradoxical upward movement can also be observed during inspiration (3). This may further hinder the action of accessory respiratory muscles and aggravate symptoms. Absence of muscle tone will often lead to progressive thinning and elevation, with permanent upward displacement of abdominal organs. When this is the case effects on mediastinal structures such as the heart can been observed, particularly on the left. Localised lung compression and atelectasis due to failed expansion can sometimes be present.

\section{Causes of iatrogenic injury}

\section{Surgery in the thorax}

During cardiac surgery the phrenic is at risk for a number of reasons including excessive retraction of the sternum, damage during mammary artery dissection, mechanical manipulation, or pericardiotomy and direct transection. It is also often attributed to cold thermal damage from iceshush used for cooling in the pericardium. It has been estimated that somewhere between $30-75 \%$ of patients undergoing cardiac surgery have some radiological evidence of unilateral phrenic nerve injury post-operatively, not all of which become symptomatic (4). The persistence of a nonspecific symptoms such as dyspnoea or orthopnoea may make recognition of the relative contribution of diaphragmatic palsy difficult to decipher from that of the background cardiac disease.

By definition, radical thymectomy requires resection of all thymic fat "from phrenic to phrenic". Close proximity of surgical manipulation therefore makes this procedure carrier significant risk of nerve injury, particularly in patients with high BMI, where visualisation or identification of the phrenic nerves may be difficult. Bilateral nerve damage is very rare but possible. In some cases when a thymic neoplasm is infiltrating the phrenic or very close to it, this may be deliberately sacrificed or heavily skeletonised to ensure tumour clearance. The effects of nerve damage are also more pronounced in patients with associated myasthenia gravis.

Phrenic nerve injury during pulmonary resection is less common but possible, particularly during dissection of mediastinal lymph nodes (station 6) on the left side (3). The risk of injury increases in presence of adhesions over the mediastinal pleura where identification or safe dissection of the phrenic nerve may be more challenging. As the phrenic nerves run subpleurally, direct tumour infiltration in the absence of preoperative evidence of diaphragmatic palsy is not very common. On the right side, the phrenic nerve runs anteriorly to the superior pulmonary vein at variable distance. Vein dissection may sometime require that the phrenic is pushed away or manipulated to remove lymph nodes and expose the vein and the surrounding structures. In addition, it is a fairly vascularised area that may require haemostasis and not infrequently localised adhesions may be found. Inattentive manoeuvres for completion of the horizontal fissure may also cause damage or transection of the nerve, particularly when instruments or mechanical staplers are advanced blindly from posteriorly. In this situation, particular care should be taken that the phrenic is visualised before firing, as it can be easily "lifted" with the inferior anvil of the stapler and taken in the staple line.

\section{Cardiac ablation techniques}

Radiofrequency energy or ultrasonic energy is used to ablate aberrant conducting pathways thought to cause arrhythmias. During this procedure the phrenic nerves can be affected by collateral damage. The right phrenic nerve is closely related to the confluence of the right superior pulmonary vein with left atrium, which is one of the target areas for ablation in atrial fibrillation procedures $(3,5)$. A 
multicentre study found the incidence of phrenic injury in $\mathrm{AF}$ ablation to be $0.4 \%$ and only resulted in permanent damage in $17 \%$ of those patients (3).

\section{Other causes}

Invasive procedures on the neck or shoulder may also occasionally produce damage to the phrenic nerve. These include surgery for thoracic outlet syndrome, cervical lymphadenectomy and also regional anaesthetic blocks for shoulder surgery (3). Radiofrequency ablation of pulmonary tumours can also risk the nerve (6).

\section{Presentation and work up}

Unilateral phrenic nerve paralysis can be clinically silent, while in other cases, and particularly in presence of underlying co-morbidities such as obesity or COPD, symptoms may be more apparent $(7,8)$. Some of these symptoms include positional dyspnoea (particularly on leaning forwards, bending down or lying flat), cough, pain, fatigue, orthopnoea, exertional dyspnoea and sleeping disorders such as sleep apnoea. Bilateral phrenic nerve injury is much more debilitating and often requires night time ventilation support (9). Gastrointestinal symptoms such as bloating, reflux, dyspepsia, constipation are also common, and cases of palpitations or cardiac arrhythmia have also been reported. Recurring chest infections may also be part of the clinical picture due to suboptimal regional lung expansion or direct compression. The reduced ability to exercise typically induces an increase in body weight and inability to lose weight.

\section{Pulmonary function testing}

Pulmonary function testing is an important feature in the work-up for diagnosis of phrenic nerve paralysis. In the case of unilateral paralysis there is often only a mild decrease in vital capacity (up to $75 \%$ predicted), typically worsening by at least $10-20 \%$ on lying down. FEV1 and FVC are less consistently affected, but a restrictive deficit pattern of variable entity may be seen $(9,10)$. As expected the findings associated with bilateral paralysis are more significant.

\section{Imaging}

A chest X-ray will show a variable degree of elevation of the affected diaphragm. Symptomatic diaphragmatic paralysis without radiological evidence of elevation at static imaging is uncommon.

Absence of movement can be confirmed with dynamic imaging techniques. Of these, ultrasound is inexpensive, easily accessible and non-invasive, however remains operator-dependent and is unable to provide real time bilateral assessment. This is particularly important for confirming the presence of paradoxical movement, due to its therapeutic implications as further on described. For this reason, fluoroscopy is the most frequently used method of assessing unilateral hemi-diaphragm paralysis. During a "sniff" test, the patient is asked to perform a rapid inspiratory manoeuvre whilst directly visualising the two hemidiaphragms. The classic sign is of a paradoxical upward motion of the diaphragm on inspiration as the contralateral diaphragm moves physiologically downwards (9). Fluoroscopy can also be used to assess bilateral weakness as both diaphragms would show an upward movement (11).

One of the main advantages ultrasound has over fluoroscopy is that it can measure the static thickness and inspiratory thickening of the diaphragm, without a large radiation dose (9). These are measured at the zone of apposition of the diaphragm. On ultrasound this can be visualised as a hypoechoic structure between two hyperechoic lines (the parietal pleura and peritoneum). At the end of expiration the diaphragm thickness can be measured. To measure the inspiratory thickening fraction (TF) of the diaphragm the thickness needs to be measured at the end of deep inspiration (total lung volume) and end of quiet expiration (function residual capacity) and these numbers subtracted from each other and expressed as a percentage of end expiratory thickness (9).

$$
T F=T_{T L C}-T_{F R C} / T_{F R C}
$$

The TF represents the strength of the diaphragm and therefore can be used as a measure of dysfunction. It has been evaluated in a critical care setting for use in predicting which patients may fail to wean from mechanical ventilation (7). An increase in thickness of $20 \%$ is reportedly the lower end of normal in healthy subjects (12).

Dynamic Magnetic Resonance provides the best possible dynamic resolution and metric assessment, however in view of the higher costs this is generally unjustified in routine clinical practice (13).

\section{Electromyography (EMG) and nerve conduction studies}

EMG is a common way of diagnosing neuromuscular 
dysfunction by describing myoelectric signals $(11,14)$. EMGs are the electrical wave form that is picked up by an electrode when an action potential is propagated along muscle fibres (11). They can be used to examine the amount and pattern of activation of the diaphragm. Diaphragmatic EMGs can be recorded using an intramuscular needle to measure the difference between the active electrode, which is placed at the lower end of the sternum, and an indifferent electrode, which is placed at the top of the sternum $(11,15)$. Disadvantages of using EMGs are that they are difficult to perform and uncomfortable for the patient, and therefore are not widely used in patient work up.

\section{Treatment options and planning}

There are three types of nerve injury, as described by Seddon (16): neurapraxia, which is an often reversible and temporary dysfunction of a nerve; axonotmesis, which is damage to neurons with preservation of supporting structures; and neurotmesis, which is complete transaction of the nerve (17). Axonotmesis and neurotmesis are often irreversible. Options for management of phrenic nerve injury depends on which type of neuronal injury has occurred and could be in the form of immediate reconstruction of the injured nerve, delayed reconstruction of the nerve or procedures to deal with its consequences such as diaphragmatic plication (6).

Figure 1 is a flow diagram giving an illustrative management algorithm for phrenic nerve injury, based on the authors' experience and current evidence in the literature.

\section{Diaphragmatic plication}

Diaphragmatic plication involves placing sutures in the atonic, paralysed hemidiaphragm with the aim of allowing it to remain static during the respiratory cycle. As diaphragmatic plication is not regaining normal movement, symptomatic improvement is achieved by removing the paradoxical movement element of the paralysed diaphragm. The decision to proceed to surgery is mainly depending on the severity of symptoms and only in their presence.

Diaphragmatic plication has been in practice since the 1920 's and the surgical approach could be either from the abdomen or the thorax. Originally the thoracic approach to diaphragmatic plication was via a thoracotomy, however advances with video-assisted surgery (VATS) have made minimally invasive approach a preferred option, with reduction of morbidity and side effects, enhanced recovery and improved functional response (18-21). This has also increased the number of patients treated or willing to explore treatments as well as their complexity. There have been a number of different ways of achieving plication described including continuous or interrupted suturing and stapling of the redundant part of the diaphragm $(22,23)$. In the technique used by the authors, the addition of carbon dioxide insufflation to a totally thoracoscopic technique has extended the ability to operate in the chest by pushing down the diaphragm. At the same time this is also safer due to better visualisation and allowing more precise suturing over the edges of a folded excess length diaphragm, with reduced risks of injury to abdominal organs (24). More recently, robotic-assisted plication has also been described (25).

When intraoperative injury is strongly suspected or ascertained, some authors have suggested that immediate plication may be beneficial (26), however the authors of this paper feel that plication of a still eutrophic and thick diaphragm has doubtful effectiveness; the effects of atrophy are likely to manifest over time as the diaphragm loses tone, thins out and overstretches. The patient therefore may well go on to develop symptoms (or indeed never develop symptoms) despite prophylactic plication. This remains therefore open to debate as the evidence is not completely convincing.

Before intervention, it is crucial that a thorough assessment of the patient history and present conditions is undertaken. This importance of this is threefold; firstly, paucisymptomatic or highly functioning patients are less likely to experience significant improvements, but remain at the same surgical risk. These are not limited to the generic possibility of inherent perioperative complications, but include the risk of potentially debilitating side effects such as pain, or the possibility of decompensation with symptom worsening. Secondly, the symptoms, albeit quite typical, remain nonspecific and could also be attributed to other underlying comorbidities. In this case, the positive effect of correction may be less predictable and be overestimated, while at the same time exposing the patient to increased risks. Thirdly, but not less important, identification and correction of risk factors should be pursued as much as possible. Patient optimisation is key to long term success and to avoid immediate morbidity. Smoking cessation, optimal management of COPD and of infective exacerbations as well as attempt to weight loss are mandatory. Screening and correction for obstructive sleep apnoea syndrome is also recommended (6).

Depending on the mechanism of injury, gradual 


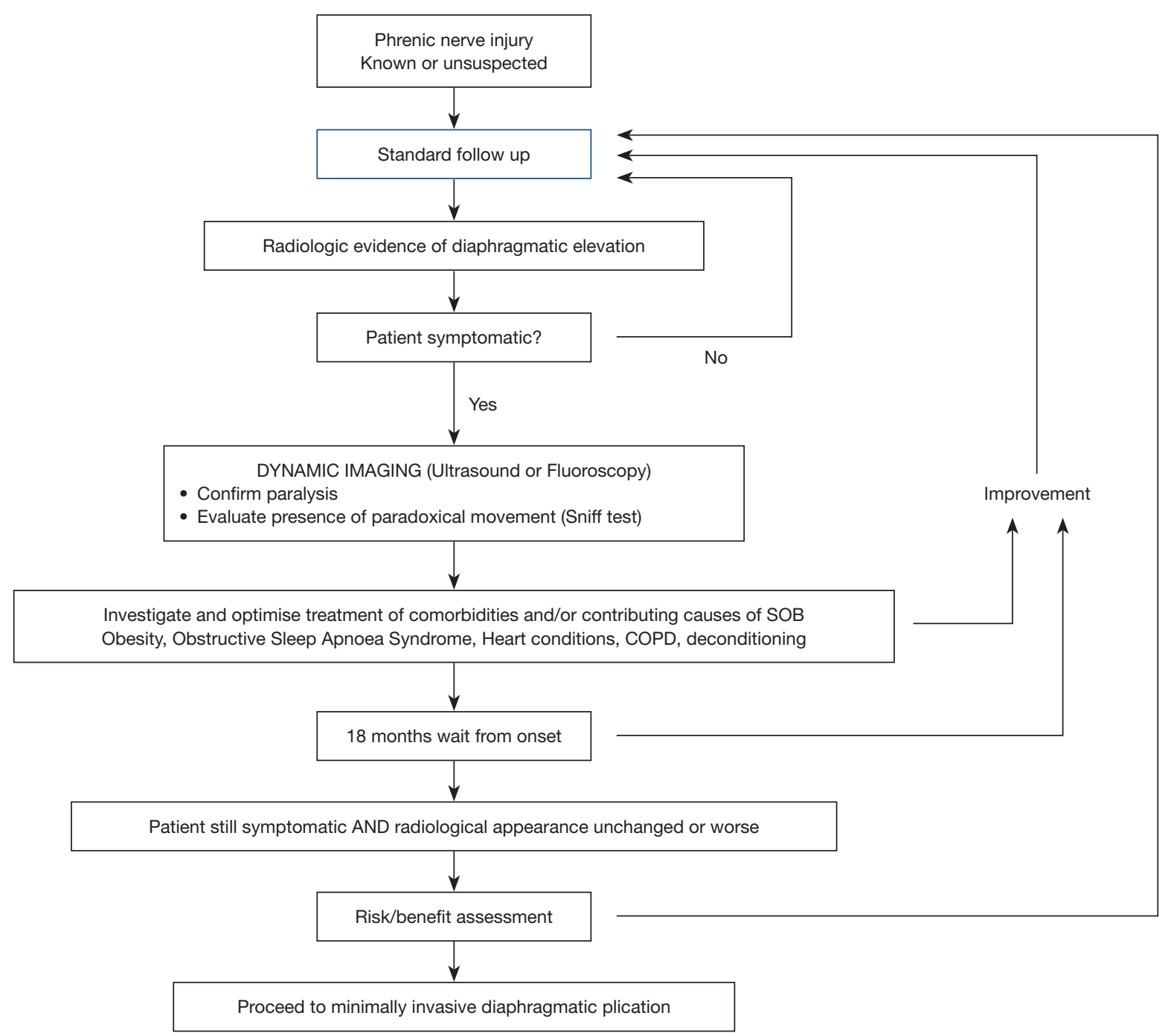

Figure 1 Algorithm for treatment.

spontaneous recovery has been observed even after many months. The authors therefore recommend against any active intervention within 18 months from the first documented radiological evidence.

Of equal importance, patient expectations should be adequately managed. As detailed below, depending on the technique used, in well selected patients a significant improvement in both subjective and objective measures has been shown, particularly with minimally invasive techniques (18-21). In a study of 25 patients undergoing plication, there was an average increase by 20 points in St George's Respiratory Questionnaire and improvement of FVC, at one month, which was also maintained at one year (27). In a review article from 2010 considering seven series of diaphragmatic plication, an improvement in FEV1 of between $7-25 \%$, with similar improvements in FVC was observed (28). However, response to treatment may also be occasionally absent, limited or unsustained, with gradual return to the baseline conditions after variable time. This is due to the diaphragm remaining atonic and thin and therefore prone to stretching and to further relaxation over time.

\section{Phrenic nerve repair techniques}

Phrenic nerve repair techniques are aimed at restoring the function of the paralysed hemi-diaphragm. Neurolysis, nerve grafting and neurotisation are techniques for nerve 
repair that have been originally utilised in peripheral nerve injuries before use on the phrenic nerve. Neurolysis is the technique of releasing the nerve from adhesions and sites of tension in order to favour spontaneous repair, whereas nerve grafting and neurotisation involve harvesting a peripheral nerve from a donor site and grafting it to the damaged nerve (17).

In a series by Kaufmen et al., a sural nerve interposition graft was used to bypass the phrenic nerve injury (17). The authors used the technique when there was either complete denervation or when neurolysis had not improved the EMG thresholds intraoperatively. Eight out of nine patients who could be evaluated post operatively experienced improvements in diaphragmatic function, including quality of life testing, pulmonary function testing and radiological imaging. The authors designed an algorithm from treatment and timing of diaphragmatic paralysis. They suggested in the presence of unilateral phrenic nerve injury the patient should undergo electrodiagnostic evaluation and if voluntary motor units were present on the EMG they could be considered for phrenic nerve reconstruction surgery, if not they would receive plication. The timing of surgery following diagnosis, similarly to plication, is $12-18$ months and requires again optimisation of weight and COPD.

A further and larger study of 180 patients from the same authors following phrenic nerve reconstruction demonstrated an improvement in nerve conduction latency (average of $23 \%$ ) and diaphragmatic motor amplitude (average of $125 \%$ ) and well as $89 \%$ of patients reporting improvement in their breathing function (15). Median follow up time was 2.7 years. The functional recovery of the diaphragm appears to take between one and two years and best results were obtained when a rehabilitation programme was also attended.

Results are certainly promising but haven't been replicated, probably due to the very specialist expertise required.

\section{Phrenic nerve pacing}

Phrenic nerve pacing requires an intact nerve to stimulate the diaphragm. The nerve is stimulated at the level of the neck or the thorax most commonly but can also be done at the level of the diaphragm (29). Its main indication is for patients who are requiring ventilatory support in the context of tetraplegia. It has limited use in unilateral phrenic nerve injury because of the difficultly in synchronising the pacemaker with spontaneous respiration (17).
In the case of high spinal cord injury at C3 to C5 level, where phrenic pacing does not work, Krieger and Krieger describe an interesting technique using a donor intercostal nerve to neurotise the phrenic nerve. They reported a series of 10 patients, where at the time of the procedure they implant a phrenic nerve pacemaker as well as connecting an intercostal nerve to transected phrenic nerve. Of the 10 transfers, 8 were able to achieve diaphragm pacing (30). This may find in the future an application in patients with iatrogenic injury as well.

\section{Conclusions}

Iatrogenic injury of the phrenic nerve can lead to distressing shortness of breath and good therapeutic options exist. Surgical intervention should only be done after an accurate assessment of the patient, and requires adequate planning and timing. Minimally invasive plication of the diaphragm remains the most widely and most successfully used technique for symptom palliation. Nerve repair and reconstruction techniques are though emerging and show great promise in these patients where motor-units are preserved.

\section{Acknowledgments}

Funding: None.

\section{Footnote}

Provenance and Peer Review: This article was commissioned by the Guest Editor (David Waller) for the series "Complications of Thoracic Surgery-aetiology, management and prevention" published in Shangbai Chest. The article was sent for external peer review organized by the Guest Editor and the editorial office.

Conflicts of Interest: All authors have completed the ICMJE uniform disclosure form (available at: http:// dx.doi.org/10.21037/shc-2019-amp-01). The series "Complications of Thoracic Surgery-aetiology, management and prevention" was commissioned by the editorial office without any funding or sponsorship. The authors have no other conflicts of interest to declare.

Ethical Statement: The authors are accountable for all aspects of the work in ensuring that questions related to the accuracy or integrity of any part of the work are 
appropriately investigated and resolved.

Open Access Statement: This is an Open Access article distributed in accordance with the Creative Commons Attribution-NonCommercial-NoDerivs 4.0 International License (CC BY-NC-ND 4.0), which permits the noncommercial replication and distribution of the article with the strict proviso that no changes or edits are made and the original work is properly cited (including links to both the formal publication through the relevant DOI and the license). See: https://creativecommons.org/licenses/by-nc-nd/4.0/.

\section{References}

1. Cummings KW, Sridhar S, Parsons MS, et al. Crosssectional Imaging Anatomy and Pathologic Conditions Affecting Thoracic Nerves. RadioGraphics 2017;37:73-92.

2. Wang J, Li J, Liu G, et al. Nerves of the Mediastinum. Thorac Surg Clin 2011;21:239-49.

3. Ko MA, Darling GE. Acquired Paralysis of the Diaphragm. Thorac Surg Clin 2009;19:501-10.

4. Efthimiou J, Butler J, Woodham C, et al. Diaphragm paralysis following cardiac surgery: Role of phrenic nerve cold injury. Ann Thorac Surg 1991;52:1005-8.

5. Smith NM, Segars L, Kauffman T, et al. Using anatomical landmark to avoid phrenic nerve injury during balloonbased procedures in atrial fibrillation patients. Surg Radiol Anat 2017;39:1369-75.

6. Auchincloss HG, Donahue DM. Prevention and Management of Nerve Injuries in Thoracic Surgery. Thorac Surg Clin 2015;25:509-15.

7. Qian Z, Yang M, Li L, et al. Ultrasound assessment of diaphragmatic dysfunction as a predictor of weaning outcome from mechanical ventilation: a systematic review and meta-analysis. BMJ Open 2018;8:e21189.

8. Caleffi-Pereira M, Pletsch-Assunção R, Cardenas L, et al. Unilateral diaphragm paralysis: a dysfunction restricted not just to one hemidiaphragm. BMC Pulm Med 2018;18:126.

9. Dubé BP, Dres M. Diaphragm Dysfunction: Diagnostic Approaches and Management Strategies. J Clin Med 2016;5:113.

10. Laroche CM, Mier AK, Moxham J, et al. Diaphragm strength in patients with recent hemidiaphragm paralysis. Thorax 1988;43:170-4.

11. ATS/ERS Statement on Respiratory Muscle Testing. Am J Crit Care 2002;166:518-624.

12. Boon AJ, Harper CJ, Ghahfarokhi LS, et al. Twodimensional ultrasound imaging of the diaphragm:
Quantitative values in normal subjects. Muscle Nerve 2013;47:884-9.

13. Kharma N. Dysfunction of the diaphragm. Curr Opin Pulm Med 2013;19:394-8.

14. Kramer C, Jordan D, Kretschmer A, et al. Electromyographic Permutation Entropy Quantifies Diaphragmatic Denervation and Reinnervation. PLoS One 2014;9:e115754.

15. Elkwood A, Brown D, Cece J, et al. Long-Term FollowUp after Phrenic Nerve Reconstruction for Diaphragmatic Paralysis: A Review of 180 Patients. J Reconstr Microsurg 2016;33:063-9.

16. Seddon H. Three Types of Nerve Injury. Brain 1943;66:237-88.

17. Kaufman MR, Elkwood AI, Rose MI, et al. Reinnervation of the Paralyzed Diaphragm: Application of Nerve Surgery Techniques Following Unilateral Phrenic Nerve Injury. Chest 2011;140:191-7.

18. Yalcinkaya I, Evman S, Lacin T, et al. Video-assisted minimally invasive diaphragmatic plication: feasibility of a recognized procedure through an uncharacteristic hybrid approach. Surg Endosc 2017;31:1772-7.

19. Mouroux J, Venissac N, Leo F, et al. Surgical Treatment of Diaphragmatic Eventration Using Video-Assisted Thoracic Surgery: A Prospective Study. Ann Thorac Surg 2005;79:308-12.

20. Demos DS, Berry MF, Backhus LM, et al. Video-assisted thoracoscopic diaphragm plication using a running suture technique is durable and effective. J Thorac Cardiovasc Surg 2017;153:1182-8.

21. Freeman RK, Van Woerkom J, Vyverberg A, et al LongTerm Follow-Up of the Functional and Physiologic Results of Diaphragm Plication in Adults With Unilateral Diaphragm Paralysis. Ann Thorac Surg 2009;88:1112-7.

22. Kara HV, Roach MJ, Balderson SS, et al. Thoracoscopic diaphragm plication. Ann Cardiothorac Surg 2015;4:573-5.

23. Kocher GJ, Zehnder A, Schmid RA. Completely Thoracoscopic Diaphragmatic Plication. World J Surg 2017;41:1019-22.

24. Dunning J. Thoracoscopic diaphragm plication. Interact Cardiovasc Thorac Surg 2015;20:689-90.

25. Taberham RJ, Raza A, Alzetani A, et al. Vats Plication of the Diaphragm a Descriptive Observational 10Year Southampton Experience. Innovations (Phila) 2017;12:398-405.

26. Beattie GW, Dunn WG, Asif M. In patients with a tumour invading the phrenic nerve does prophylactic diaphragm plication improve postoperative lung function. Interact 
Cardiovasc Thorac Surg 2016;23:454-8.

27. Groth SS, Rueth NM, Kast T, et al. Laparoscopic diaphragmatic plication for diaphragmatic paralysis and eventration: An objective evaluation of shortterm and midterm results. J Thorac Cardiovasc Surg 2010;139:1452-6.

28. Groth SS, Andrade RS. Diaphragm Plication for Eventration or Paralysis: A Review of the Literature. Ann Thorac Surg 2010;89:S2146-50.

doi: 10.21037/shc-2019-amp-01

Cite this article as: Whiteley J, Shoeib M, Bilancia R. Iatrogenic phrenic nerve palsy. Shanghai Chest 2021;5:27.
29. Marion D. UpToDate [Internet]. Uptodate.com 2020 [cited 12 January 2020]. Available online: https://www. uptodate.com/contents/pacing-the-diaphragm-patientselection-evaluation-implantation-and-complications

30. Krieger LM, Krieger AJ. The Intercostal to Phrenic Nerve Transfer: An Effective Means of Reanimating the Diaphragm in Patients with High Cervical Spine Injury. Plast Reconstr Surg 2000;105:1255-61. 